\title{
Entry, Competition, and Regulation in Cognitive Radio Scenarios: A Simple Game Theory Model
}

\author{
Luis Guijarro, Vicent Pla, Jose R. Vidal, \\ and Jorge Martinez-Bauset \\ Departamento de Comunicaciones, Universitat Politècnica de València, 46022 València, Spain \\ Correspondence should be addressed to Luis Guijarro, lguijar@dcom.upv.es
}

Received 2 October 2012; Accepted 7 November 2012

Academic Editor: Matjaz Perc

Copyright (c) 2012 Luis Guijarro et al. This is an open access article distributed under the Creative Commons Attribution License, which permits unrestricted use, distribution, and reproduction in any medium, provided the original work is properly cited.

Spectrum management based on private commons is argued to be a realistic scenario for cognitive radio deployment within the current mobile market structure. A scenario is proposed where a secondary entrant operator leases spectrum from a primary incumbent operator. The secondary operator innovates incorporating cognitive radio technology, and it competes in quality of service and price against the primary operator in order to provide service to users. We aim to assess which benefit users get from the entry of secondary operators in the market. A game theory-based model for analyzing both the competition between operators and the subscription decision by users is proposed. We conclude that an entrant operator adopting an innovative technology is better off entering the market, and that a regulatory authority should intervene first allowing the entrant operator to enter the market and then setting a maximum amount of spectrum leased. This regulatory intervention is justified in terms of users utility and social welfare.

\section{Introduction}

The traditional approach to spectrum management, widely known as command- and-control, has been highly prescriptive, since regulators decide not only the use of a particular band, but also which users are allowed to transmit in the band. Although the approach can result in highly technical efficiency of spectrum use, under current circumstances of spectrum scarcity, there has been much evidence that suggests that regulators are failing to maximize value.

Alternative approaches to command-and-control have been proposed and tested for the two last decades. A range of approaches fall under the general name of market mechanisms. The essential idea is to allow pricing mechanisms to act as an incentive for holders of spectrum to optimize their use, buying more if their business case can justify it, selling spectrum if they have in excess, and adopting technologies that can use spectrum 
more efficiently where economically viable. Economic theory suggests that in a market which is performing well, this will lead to a division of spectrum that maximizes economic value.

The simplest of the market instruments to adopt is probably the use of auctions as a mechanism for distributing spectrum. Interest in using auctions to assign frequency rights increased markedly around the world during the 1990s. But auctions on their own still "freeze" the assignment of spectrum. Auctions need to be accompanied by mechanisms to trade and change the use of spectrum as market conditions change, and new services become available. Secondary trading (aka. spectrum trading) is the trading of spectrum rights after the primary assignment. Proponents of spectrum trading argue that the design of markets intended to facilitate transactions should result in economically efficient outcomes. Furthermore, spectrum trading is made much more powerful when it is combined with policies aimed at promoting flexibility in use, as far as services and technologies are concerned.

Another alternative to command-and-control is the commons approach. It is based on the argument that radio spectrum is a public resource that should be equitably and fairly accessible to everyone without undue government regulations. The commons model has some variants. In the purest variant, no entity has exclusive license to the spectrum band. As such, anyone can own any number of devices operating in such a band-which is the case of the current ISM $(2.4 \mathrm{GHz})$ band.

A new concept within the commons model was introduced by the FCC in 2004 which is called private commons [1]. The concept allows new agents to access the spectrum owned by a license holder, where the responsibility of setting rules for access by devices not participating in primary use of the license, and enforcement of those rules is entirely with the license holder. Under private commons, the license holder maintains a customer relationship with the new agents and may charge a transaction fee for the relationship.

Following a similar evolution, technology has made progresses which have matched the evolution in spectrum management described above. We would like to focus on cognitive radio. This technology aims at allowing aggressive and opportunistic exploitation of white spaces or spectrum "gaps" in spatiotemporal domain. In its simplest operational model, given a primary spectrum user with an exclusive use license to a spectrum band, a homogeneous population of secondary users continuously performs spectrum sensing to estimate whether a spectrum access opportunity is available, and when it is available, the secondary users contend or coordinate their transmissions, until an onset of primary transmission is detected. Then the secondary devices must cease transmission and "vacate" the spectrum.

Although the cognitive radio technology is promising, there are a number of questions which require investigation. We would like to draw attention to the possibility that the transmissions of a large number of secondary users can eventually be free of charge in a band that the primary operator has paid to acquire. We argue that this possibility seems unfeasible and that the deployment of cognitive radio networks will only be viable by implementing a payout from the secondary users to the primary operator.

We agree with [2] that private commons may be a viable market option for cognitive radio networks. On the one hand, such a model may be attractive to secondary users, who may find guarantees provided by the licensed band attractive as an alternative to unlicensed bands that may be overcrowded. On the other hand, it allows an exclusive use license holder to "get adventurous" to aggressively use its spectrum, potentially for increased revenue stream via per user periodic transactions-a form of a spot secondary market. Reference [3] actually proposes this scheme in order to realize Wi-Fi-like internet access operating on 
whitespaces in the licensed spectrum and using cognitive radio technology, which is being called Wi-Fi 2.0.

In the context of the above discussion, this paper analyzes a realization of the private commons model where the secondary users implement cognitive radio technology and are serviced by a mobile communications operator. The "secondary operator" coordinates the access to spectrum "gaps" by the secondary users, and it conducts the associated spectrum trading on a real-time or quasi-real-time basis with the license holder or "primary operator".

Many papers can be found which analyze spectrum trading within the context of cognitive radio networks. Reference [4] analyzes several secondary operators which request spectrum to a license holder and which compete in terms of the requested spectrum size. The license holder charges the operators according to a pricing function. A simple noncooperative one-shot game model is proposed to find the equilibrium. More complex models can be found in $[5,6]$, where two secondary operators lease spectrum from a license holder and compete with each other in order to provide service to secondary users. Each secondary operator should decide first upon the amount of spectrum leased and second upon the price charged for the service, anticipating the users subscription decision. The users behavior may be explicitly modeled through individual utility functions [6] or in an aggregated manner by means of a demand function [5]. In both papers, a multiple-stage dynamic game is proposed to find the equilibrium.

The above models assume that secondary operators are the only ones which compete to provide service to the users. In other words, that the market is created from scratch when the secondary operators start to provide service. We argue that this assumption is unrealistic, and that any realistic model should explicitly take into account the market legacy, that is, the presence of an incumbent operator. Furthermore, within the framework of private commons, this incumbent operator is the license holder which takes responsibility over the private commons operation. This paper therefore analyzes a private commons scenario where the primary operator is the incumbent operator and where a secondary operator intends to enter the market. We believe that this scenario is the only feasible model for private commons in cognitive radio networks.

Finally, this paper models in an explicit manner the utility received by the users when subscribing to service. By explicit, we mean that no simplification is assumed for the aggregated user demand, for example [5]. Instead, an expression for the individual user utility is proposed. In this expression, we introduce some basic technology-related parameters which contribute to the transfer rate available for each user. This transfer rate is the main indicator for the quality of service received by the users. A similar approach in the modeling of the user utility as ours can be found in [7], which models the interaction between a mobile operator and a mobile virtual operator, and where the resource is measured in terms of available time slots.

Our contribution is the introduction and analysis of a model representing the economic interactions between the primary operator $(\mathrm{PO})$, the secondary operator $(\mathrm{SO})$, and the users, within the context of a private commons model and in general investigating the viability of such a system. We design a two-level game where at the higher level the operators play on the price, they will propose to users; at the lower level users distribute themselves between operators depending on price and quality of service (QoS). The offered QoS also depends on the number of customers, hence a fixed point equation to solve arises. We assume a number of users large enough, so that the action of a single user will have a negligible impact on the QoS received by the others. The game is solved by backward induction, meaning that at a given level players act strategically by anticipating the solution of the game at the lower 
level. For fixed prices set by providers, we explicitly characterize the solution at the lower game level as a Wardrop equilibrium, a concept coming from transportation theory [8]. Nash equilibrium is used to determine the solution of the pricing game between providers trying to maximize revenue, when the amount of leased spectrum and the leasing cost are fixed. Wardrop equilibrium, introduced as far back as 1952, forms the game theoretic foundation of the investigation of population dynamics under evolution or learning behaviors. Since the mid seventies, evolutionary game theory has provided an extension and a solid mathematical background to the intuition of stability present from the inception. See [9] for a discussion on the relationship of Wardrop equilibrium and the Evolutionary Stable Strategy concept, and see $[10,11]$ for more recent advances in evolutionary game theory.

The rest of this paper is organized as follows. Next section describes the scenario under analysis, and it develops the model, specifying each phase comprising the game, namely, the subscription phase and the competition phase. Section 3 describes the procedure followed to evaluate the different equilibria that may arise in the game and shows the results obtained, discussing the effect of the different parameters on the game equilibrium and assessing the outcome from the point of view of the welfare. And finally, Section 4 draws some conclusions.

\section{Model and Analysis}

The model we will analyze is depicted in Figure 1. It is made of the following:

(i) one $\mathrm{PO}$ and one $\mathrm{SO}$ which compete in the service provision to the users;

(ii) $n$ users that are assumed to have dual terminals, so that no technology-related switching costs are incurred when making the subscription decision.

The PO leases an amount of $b \mathrm{kHz}$ to the SO, keeping for itself the rest, up to the total amount of its licensed $W \mathrm{kHz}$. The SO pays for that amount a price $p$ m.u. per kHz. (m.u. $=$ monetary units). Both the price $p$ and the leased spectrum amount $b$ are assumed to be exogenously determined.

Users pay a subscription fee to the operator they have decided to subscripe to. A user would pay $p_{p}$ m.u. if she subscribed to PO service, or $p_{s}$ m.u. if she subscribed to SO service. All three prices $p, p_{p}$, and $p_{s}$ are computed with respect to the same time period.

We assume that the operators compete $a ̀$ la Bertrand [12]; that is, they are playing a one-shot simultaneous game where $\mathrm{PO}$ and $\mathrm{SO}$ strategies are $p_{p}$ and $p_{s}$, respectively. The equilibrium concept here is the Nash equilibrium.

Finally, each user will subscribe to the service providing the highest utility, which will be shown to depend on the quality of service and on the price. The equilibrium notion is the so-called Wardrop equilibrium $[8,13]$. Wardrop equilibrium was proposed by Wardrop, an English transport analyst, in 1952, as a rule to solve the traffic assignment problem, which concerns the selection of routes between origins and destinations in transportation networks. We are specifically interested in Wardrop's first principle, which says that:

"the journey times on all routes actually used are equal, and less than those which would be experienced by a single vehicle on any unused route."

This principle postulates that each vehicle seeks in a noncooperative manner to minimize its journey time, which is similar to the Nash equilibrium. Additionally, the Wardrop's first principle assumes that the population of vehicles is infinite, so that 


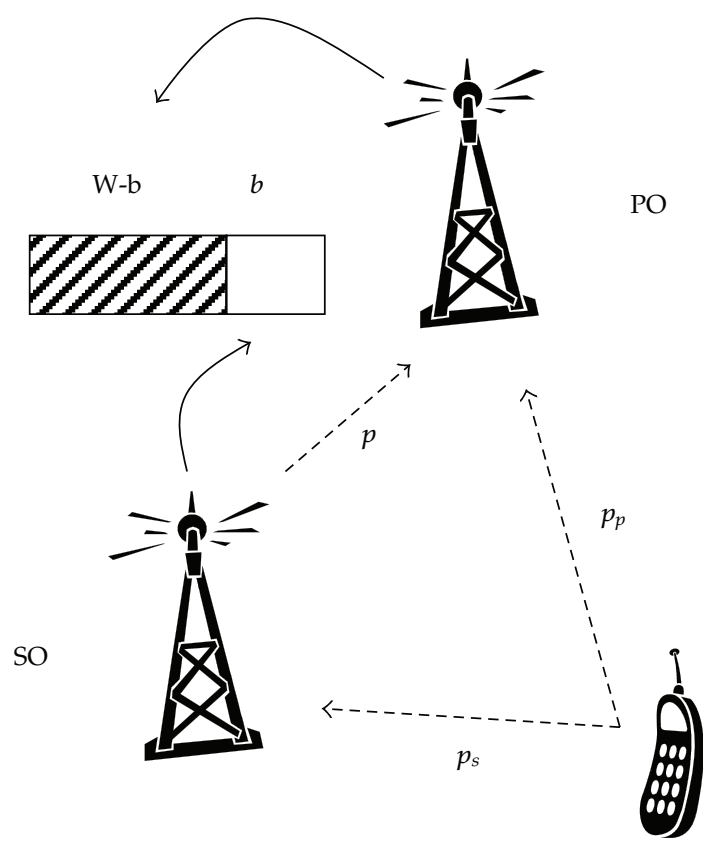

Figure 1: Payment flows and use of resources by the different agents.

the contribution to journey times by any individual vehicle is zero. This allows to postulate an identical journey times equilibrium.

Applied to our model, in the Wardrop equilibrium the users are indifferent between choosing one operator or the other one, and therefore no user has an incentive to switch, and assuming that the number of users $(n)$ is high enough, the individual subscription decision of each user will not affect the utility perceived by the rest. That will imply that the utility received at the Wardrop equilibrium by every user will be the same regardless of the operator she subscribes to.

Taking into account both the operators and users behaviors, the strategic interaction between the two operators and the $n$ users is modeled using noncooperative game theory. Specifically, we use a two-level multileader-follower game, which comprises two games:

(1) Price Competition Game:

The PO offers a subscription price $p_{p}$, and the $\mathrm{SO}$ offers $p_{s}$.

(2) Subscription Game:

Each user chooses which operator to subscribe to.

A standard way to analyze this sort of games is by means of backward induction: the price competition game is played knowing-anticipating — what would be the outcome of the subscription game, which is played later. We now show how the outcomes of those games can be computed. 


\subsection{Subscription Game: Second Phase}

In this phase, a pair of values $b$ and $p$ has been fixed, and the prices $p_{p}$ and $p_{s}$ have been announced.

The utility that the users receive from each operator depends on two factors.

\section{Quality of Service}

Each operator exploits, during each subscription period, an amount of spectrum $b$ for the $\mathrm{SO}$ and $W-b$ for the PO. Furthermore, each operator is assumed to use a different technology, which results in different levels of spectral efficiency: $k^{(p)}$ and $k^{(s)}$ are the spectral efficiencies for the $\mathrm{PO}$ and the $\mathrm{SO}$, respectively. Both the modulation schemes and the medium access control mechanisms are modeled by $k^{(p)}$ and $k^{(s)}$. We assume that $k^{(p)}<k^{(s)}$. Bearing in mind the above two issues, the product of the spectrum by the spectral efficiency for each operator, divided by the number of users which subscribe to it, will give the transfer rate that is offered to each user [14]. We propose to use this transfer rate as the quality factor $\left(Q_{p}\right.$ and $Q_{s}$ ) contributing to the user utility, through an increasing concave (logarithmic) function [15]. Specifically,

$$
\begin{gathered}
Q_{p}=\log \left(k^{(p)} \frac{W-b}{n_{p}}\right), \\
Q_{s}=\log \left(k^{(s)} \frac{b}{n_{s}}\right),
\end{gathered}
$$

where $n_{p}$ (resp., $n_{s}$ ) is the number of users subscribing to the PO, respectively, the SO.

Price

The higher the subscription price, the lower the user utility. We consider quasi-linear user utility functions: the utility is the difference between the value of the service and the price paid.

Based on the above discussion, the expressions for that user utility if subscribing to the $\mathrm{PO}$ or the $\mathrm{SO}$ are, respectively,

$$
\begin{gathered}
U_{p}=Q_{p}-p_{p}=\log \left(k^{(p)} \frac{W-b}{n_{p}}\right)-p_{p}, \\
U_{s}=Q_{s}-p_{s}=\log \left(k^{(s)} \frac{b}{n_{s}}\right)-p_{s} .
\end{gathered}
$$

A selfish user will subscribe to the service provided by the PO rather than the SO if $U_{p}>U_{s}$, that is,

$$
\log \left(k^{(p)} \frac{W-b}{n_{p}}\right)-p_{p}>\log \left(k^{(s)} \frac{b}{n_{s}}\right)-p_{s}
$$

and reciprocally. 
As stated above, at a user (Wardrop) equilibrium, the users should spread between the operators $\mathrm{PO}$ and $\mathrm{SO}$ in such a way that no user has an incentive to switch to the competing operator. For this to happen, $U_{p}=U_{s}$ must hold for every subscriber or equivalently:

$$
\log \left(k^{(p)} \frac{W-b}{n_{p}^{*}}\right)-p_{p}=\log \left(k^{(s)} \frac{b}{n_{s}^{*}}\right)-p_{s},
$$

where the asterisk stands for equilibrium values.

Given that we assume that all users subscribe service, and the following restriction holds

$$
n_{p}+n_{s}=n
$$

Let $\alpha$ denote the fraction of subscribers that subscribe to PO, and $1-\alpha$ to SO. Then $n_{p}$ and $n_{s}$ can be expressed as functions of $\alpha$ as follows:

$$
n_{p}=\alpha n, \quad n_{s}=(1-\alpha) n
$$

\subsection{Price Competition Game: First Phase}

In this phase, a pair of values $b$ and $p$ has been fixed, and each operator chooses its pricing strategy so as to maximize its profits. Each operator does not know the strategy chosen by its competitor, but common knowledge of the strategies available to them and the corresponding profits is assumed. The outcome of the subscription game is assumed to be anticipated by both operators and taken into account in the pricing decisions.

The profits of the $\mathrm{PO}$ and the $\mathrm{SO}$ can be expressed, respectively, as

$$
\begin{aligned}
& \Pi_{p}=n_{p} \cdot p_{p}+p \cdot b-C_{p}, \\
& \Pi_{s}=n_{s} \cdot p_{s}-p \cdot b-C_{s},
\end{aligned}
$$

where $C_{p}$ and $C_{s}$ are the operating costs born by the $\mathrm{PO}$ and the $\mathrm{SO}$, respectively.

When solving the equilibrium equations for the first and the second phase, $n_{p}^{*}$ and $n_{s}^{*}$ may be expressed as functions of $p_{p}$ and $p_{s}$, so that operator profits are functions of $p_{p}$ and $p_{s}$ only:

$$
\Pi_{p}=\Pi_{p}\left(p_{p}, p_{s}\right), \quad \Pi_{p}=\Pi_{s}\left(p_{p}, p_{s}\right) .
$$

Now, turning our attention to the pricing game, the equilibrium strategies $p_{p}^{*}$ and $p_{s}^{*}$ are given by the Nash equilibrium conditions [12]:

$$
\begin{aligned}
& \Pi_{p}\left(p_{p}^{*}, p_{s}^{*}\right) \geq \Pi_{p}\left(p_{p}, p_{s}^{*}\right), \quad \forall p_{p}, \\
& \Pi_{s}\left(p_{p}^{*}, p_{s}^{*}\right) \geq \Pi_{s}\left(p_{p}^{*}, p_{s}\right), \quad \forall p_{s},
\end{aligned}
$$

meaning that no operator can unilaterally increase its profits by a price change. 
Under the assumption that the partial derivatives of $\Pi_{p}$ and of $\Pi_{s}$ with respect to $p_{p}$ and $p_{s}$ exist, the Nash equilibrium can be determined by looking at the solutions of the firstorder conditions:

$$
\begin{gathered}
0=\frac{\partial \Pi_{p}\left(p_{p}^{*}, p_{s}^{*}\right)}{\partial p_{p}}=n\left(\alpha^{*}+p_{p}^{*} \frac{\partial \alpha^{*}}{\partial p_{p}}\right), \\
0=\frac{\partial \Pi_{s}\left(p_{p}^{*}, p_{s}^{*}\right)}{\partial p_{s}}=n\left(\left(1-\alpha^{*}\right)-p_{s}^{*} \frac{\partial \alpha^{*}}{\partial p_{s}}\right) .
\end{gathered}
$$

To obtain the expression of the partial derivatives of $\alpha^{*}$ with respect to $p_{p}$ and $p_{s}$, we proceed as follows. From (2.4), (2.5), and (2.6) we obtain

$$
p_{p}-p_{s}=\log \left(\frac{k^{(p)}(W-b)}{k^{(s)} b}\right)-\log \alpha^{*}+\log \left(1-\alpha^{*}\right),
$$

which can be differentiated with respect to $p_{p}$ and $p_{s}$ and gives

$$
\frac{\partial \alpha^{*}}{\partial p_{p}}=-\frac{\partial \alpha^{*}}{\partial p_{s}}=-\alpha^{*}\left(1-\alpha^{*}\right)
$$
of $\alpha^{*}$.

If thsse above expression is inserted in (2.10), $p_{p}^{*}$ and $p_{s}^{*}$ can be expressed as functions

$$
\begin{gathered}
p_{p}^{*}=\frac{1}{1-\alpha^{*}}, \\
p_{s}^{*}=\frac{1}{1-\left(1-\alpha^{*}\right)} .
\end{gathered}
$$

Finally, substituting (2.13) in (2.4), the following equation is obtained for $\alpha^{*}$, the fraction of subscribers selecting PO at the equilibrium as follows:

$$
\frac{1}{1-\alpha^{*}}=\log \left(1-\alpha^{*}\right)+\frac{1}{1-\left(1-\alpha^{*}\right)}+\log \left(\frac{k^{(p)}(W-b)}{k^{(s)} b}\right) \text {. }
$$

Note that the function $f(x)=\log x+1 /(1-x)$ is continuous, increasing for $x>0$ and that $\lim _{x \rightarrow 0}=-\infty$. We can then infer that there exists a unique value of $\alpha^{*} \in(0,1)$ which satisfies (2.14).

\section{Results and Discussion}

From the equations of the preceding section, given parameters $n, W, b, k^{(p)}, k^{(s)}$, and $p$, we model the competition between $\mathrm{PO}$ and $\mathrm{SO}$ for the service provision to users with dual 
terminals as a multileader-follower game, which results in Wardrop equilibrium market share $\alpha^{*}$ from solving (2.14), and Nash equilibrium prices $p_{p}^{*}$ and $p_{s}^{*}$ obtained from (2.13). Asterisks are hereafter removed for the sake of simplicity.

The values for the parameters, if not stated otherwise, are the following ones: $n=$ 10000 user, $W=100 \mathrm{kHz}, C_{p}=20 \mathrm{~m}$. u., $C_{s}=10 \mathrm{~m} . u$.,$k^{(p)}=1 \mathrm{bit} / \mathrm{s} / \mathrm{Hz}$, and $k^{(s)}=2 \mathrm{bit} / \mathrm{s} / \mathrm{Hz}$. A value of $p=120$ m.u. $/ \mathrm{kHz}$ has been chosen.

In order to assess the equilibrium, we have solved the equations and computed the following indicators:

(i) PO market share $\alpha$;

(ii) prices $p_{p}$ and $p_{s}$;

(iii) user utilities $U_{p}=U_{s}$;

(iv) operator profits $\Pi_{p}$ and $\Pi_{s}$;

(v) the Price of Anarchy (PoA).

The PoA is related to the concept of social welfare [16] or total surplus [17], which is computed as the sum of the utilities of all agents in the systems (i.e., users and operators). Using the notation of our model, social welfare SW can be expressed as

$$
\mathrm{SW}=n_{p} \cdot U_{p}+n_{s} \cdot U_{s}+\Pi_{p}+\Pi_{s} .
$$

It is well known in game theory that agent selfishness, such as in a Nash equilibrium, does not lead in general to a socially efficient situation, that is, yielding maximum social welfare. As a measure of the loss of efficiency due to the divergence of user interests, we use the Price of Anarchy [18], which we defined as the quotient between the maximum value of the social welfare and the social welfare obtained at the Nash equilibrium. This quotient is becoming increasingly popular for measuring how inefficient a system is when independent agents are operating in a decentralized manner; see, for instance, its application to resource allocation in $[19,20]$.

In the following paragraphs, we present the results of two experiments.

\subsection{On the Value of the Leased Spectrum}

In Figures 2 and 3, we show the effect of varying the amount $b$ of leased spectrum.

We point out that, first, as $b$ increases, the user utility reaches a maximum at $b / W \approx$ 0.45 , while the maximum social welfare $(\mathrm{PoA}=1)$ is reached at $b / W \approx 0.35$. Second, as $b$ increases from $b=0$, the PO loses users, and it is forced to lower $p_{p} ; \Pi_{p}$ decreases, and although it increases for higher values of $b$, it does not reach the initial value, and third, the SO stoles users from the $\mathrm{PO}$, and it is able to raise $p_{s}$. Thus, $\Pi_{s}$ increases as $b$ increases.

The above observations mean that:

(i) the PO does not have any incentive to lease spectrum. This fact suggests that the incumbent operator should be forced to lease a minimum amount of spectrum in order to reach the user or social optima;

(ii) once in the vicinity of these optimal points, both the $\mathrm{PO}$ and the SO prefer $b / W$ to be as large as possible, that is, equal to 1 . This behavior suggests that a maximum amount of leased spectrum should be set. 


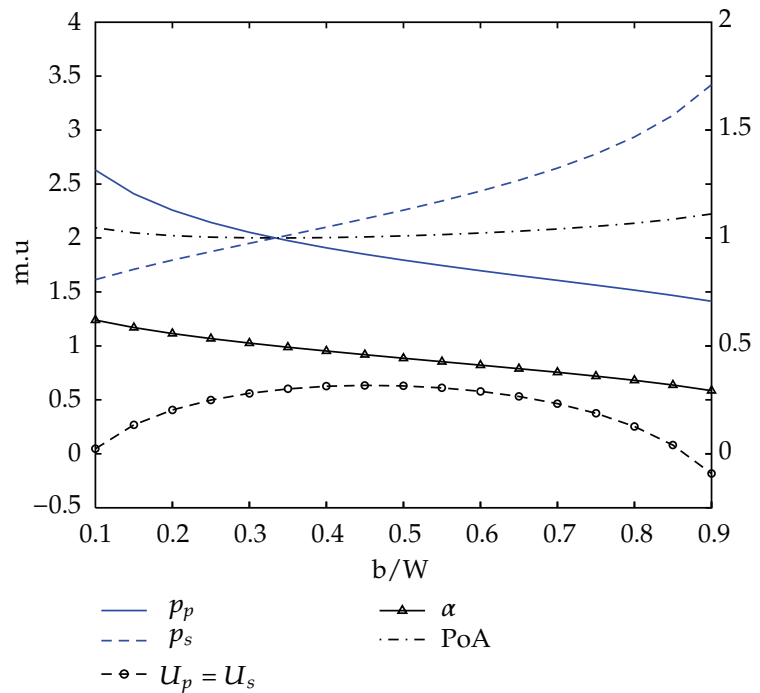

Figure 2: Effect of variation of $b / W$ on $\alpha$, prices, utilities, and PoA.

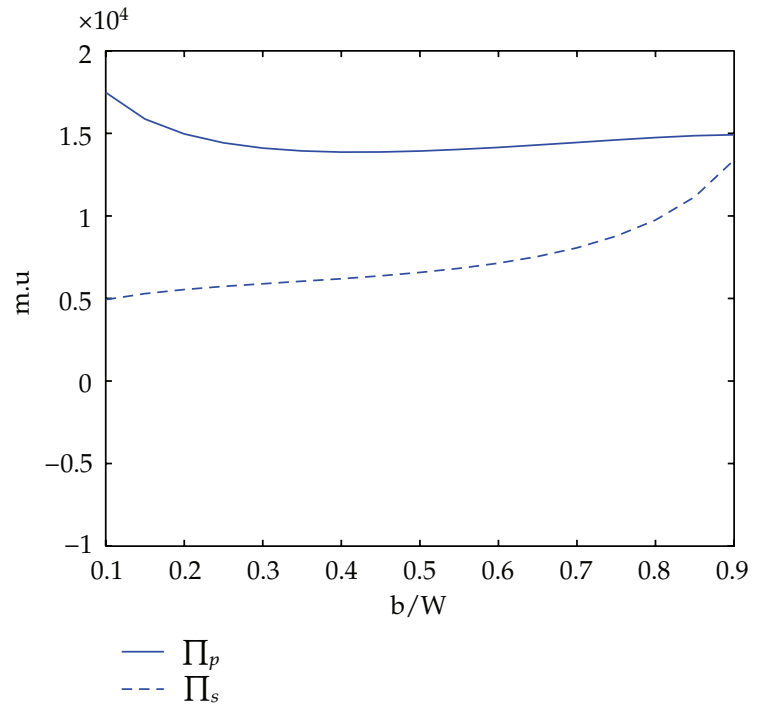

Figure 3: Effect of variation of $b / W$ on profits.

We can conclude, therefore, that a regulatory intervention case is justified, where both a minimum and a maximum values for leased spectrum should be set by a regulatory authority.

\subsection{Spectral Efficiency}

In Figures 4 and 5 , we show the effect of varying the spectral efficiency $k^{(s)}$. The value for $b$ is fixed at $b / W=0.2$. 


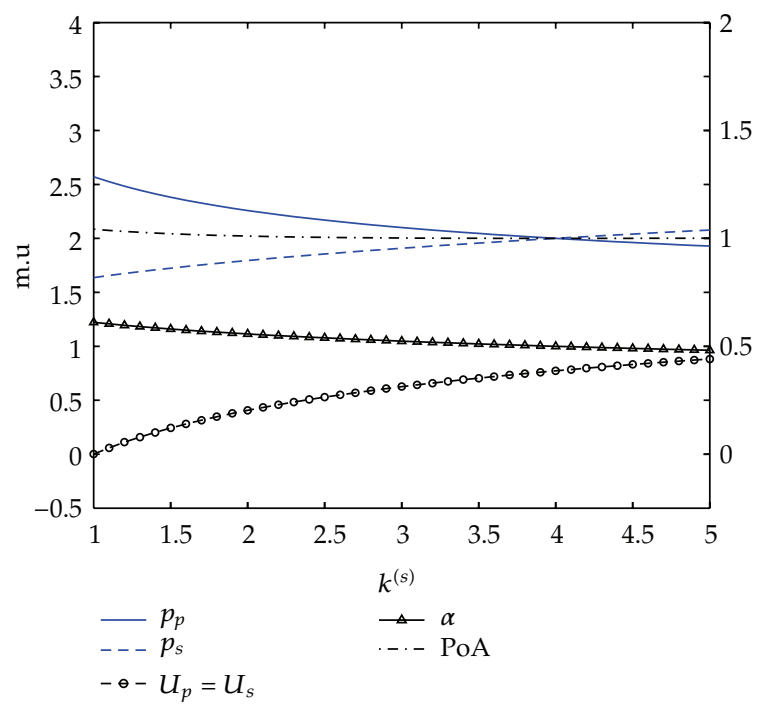

Figure 4: Effect of variation of $k^{(s)}$ on $\alpha$, prices, utilities, and PoA.

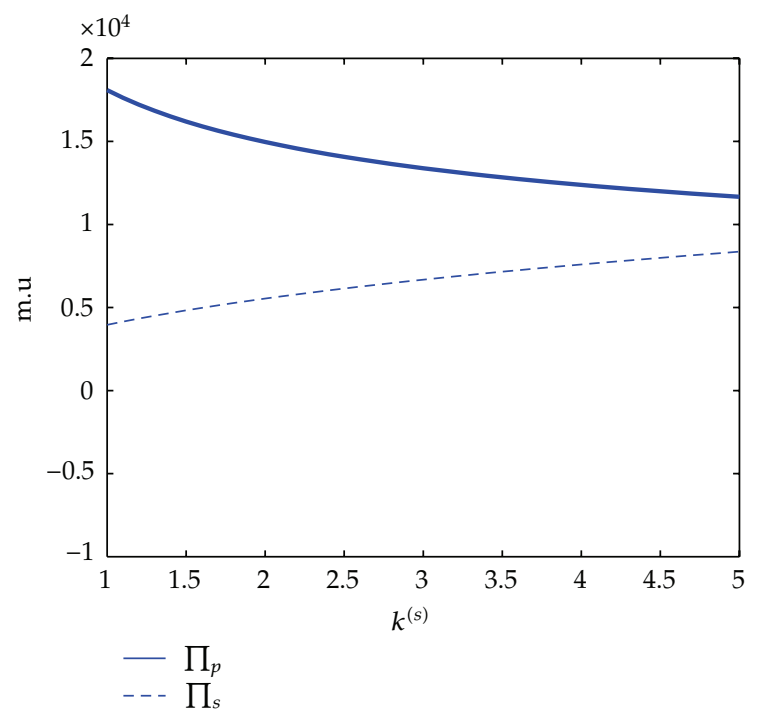

Figure 5: Effect of variation of $k^{(s)}$ on profits.

We point out that, as $k^{(s)}$ increases with respect to $k^{(p)}=1$, the user utility improves, and the social welfare keeps high; the PoA is slightly greater than 1 and approaching the unity. From the point of view of the operators, the PO loses users, and it is forced to lower $p_{p}$ and reduces its profits $\Pi_{p}$, while the SO increases its market share, and it is able to raise $p_{s}$ and increases its profits $\Pi_{s}$.

We can conclude that the improvement in the QoS that the SO achieves through technology innovation $\left(k^{(s)}>k^{(p)}\right)$ causes an improvement not only on the SO profits but also on the utility received by all users. 


\section{Conclusions}

We have proposed a realistic scenario for mobile communications service deployment based on the introduction of cognitive radio technology. The cognitive radio technology is adopted by an entrant operator, which is then technologically more efficient than the incumbent operator. The incumbent operator owns a spectrum license, and it leases spectrum to the entrant operator.

We have modeled the economical interaction between the incumbent and the entrant, and we have analyzed the effects of the entry from the point of view of the mobile communications users, from that of the incumbent, from that of the entrant, and from that of the general interest.

The conclusions reached have been the following ones.

(1) There is indeed a business case for the entrant, which gets higher profits the larger the fraction of spectrum leased is.

(2) The technological innovation adopted by the entrant, which is modeled by the spectral efficiency, is beneficial for the entrant.

(3) All users improve their situation by the facts that some spectrum is leased to the entrant (up to a fraction of the total available amount) and that the entrant innovates.

(4) Regulatory intervention is justified by users utility and social welfare arguments.

In a future work, we plan to extend our analysis in three ways. First, to allow both operators to bargain over the price and quantity of leased spectrum, so that neither $b$ nor $p$ are set exogenously. Second, to model the user willingness to pay, so that some users may decide to subscribe to neither PO nor SO. Thirdly, to gain better insight into the interaction between the users, progressing away from the simplifying assumptions of Wardrop equilibrium. A promising line of analysis is evolutionary game theory, which would incorporate evolutionary dynamics so as to model the behavior of the users in a more realistic way. More advanced approaches, such as coevolutionary game theory, which would assist in modeling how the interaction between users change in time, may be fruitful [21].

\section{Acknowledgment}

This work was supported by Spanish government through project TIN2010-21378-C02-02.

\section{References}

[1] FCC, "Promoting Efficient Use of Spectrum Through Elimination of Barriers to the Development of Secondary Markets," no. FCC 04-167, 2004.

[2] M. M. Buddhikot, "Understanding dynamic spectrum access: Models, taxonomy and challenges," in Proceedings of the 2nd IEEE International Symposium on New Frontiers in Dynamic Spectrum Access Networks, pp. 649-663, April 2007.

[3] H. Kim and K. Shin, "Understanding Wi-Fi 2.0: from the economical perspective of wireless service providers," IEEE Wireless Communications, vol. 17, no. 4, pp. 41-46, 2010.

[4] D. Niyato and E. Hossain, "Competitive spectrum sharing in cognitive radio networks: a dynamic game approach," IEEE Transactions on Wireless Communications, vol. 7, no. 7, pp. 2651-2660, 2008. 
[5] J. Jia and Q. Zhang, "Competitions and dynamics of duopoly wireless service providers in dynamic spectrum market," in Proceedings of the 9th ACM International Symposium on Mobile Ad Hoc Networking and Computing 2008 (MobiHoc'08), pp. 313-322, May 2008.

[6] L. Duan, J. Huang, and B. Shou, "Competition with dynamic spectrum leasing," in Proceedings of the IEEE Symposium on New Frontiers in Dynamic Spectrum (DySPAN '10), April 2010.

[7] H. Le Cadre and M. Bouhtou, "Modelling MNO and MVNOs dynamic interconnection relations: is cooperative content investment profitable for both providers?" Telecommunication Systems, vol. 51, no. 2, pp. 193-217, 2012.

[8] J. Wardrop, "Some theoretical aspects of road traffic research," Proceedings of the Institute of Civil Engineers, vol. 1, pp. 325-378, 1952.

[9] P. Bernhard, "ESS, population games, replicator dynamics: dynamics and games if not dynamic games," in Advances in Dynamic Games, vol. 11, pp. 291-311, 2011.

[10] Z. Wang, A. Szolnoki, and M. Perc, "Evolution of public cooperation on interdependent networks: the impact of biased utility functions," Europhysics Letters, vol. 97, no. 4, p. 48001, 2012.

[11] A. Szolnoki and M. Perc, "Resolving social dilemmas on evolving random networks," Europhysics Letters, vol. 86, no. 3, p. 30007, 2009.

[12] M. Osborne, An Introduction to Game Theory, Oxford University Press, New York, NY, USA, 2004.

[13] E. Altman, T. Boulogne, R. El-Azouzi, T. Jiménez, and L. Wynter, "A survey on networking games in telecommunications," Computers E Operations Research, vol. 33, no. 2, pp. 286-311, 2006.

[14] D. Niyato and E. Hossain, "Competitive pricing for spectrum sharing in cognitive radio networks: dynamic game, inefficiency of nash equilibrium, and collusion," IEEE Journal on Selected Areas in Communications, vol. 26, no. 1, pp. 192-202, 2008.

[15] P. Reichl, B. Tuffin, and R. Schatz, "Logarithmic laws in service quality perception: where microeconomics meets psychophysics and quality of experience," Telecommunication Systems. In press.

[16] P. Maillé and B. Tuffin, "Analysis of price competition in a slotted resource allocation game," in Proceedings of the 27th IEEE Communications Society Conference on Computer Communications (INFOCOM '08), pp. 1561-1569, April 2008.

[17] H. Varian, Microeconomic Analysis, Norton, New York, NY, USA, 1992.

[18] K. Papadimitriou and E. Koutsoupias, "Worst-case equilibria," in Proceedings of the Symposium on Theoretical Aspects of Computer Science (STACS '99), pp. 404-413, 1999.

[19] E. Altman, U. Ayesta, and B. Prabhu, "Load balancing in processor sharing systems," Telecommunication Systems, vol. 47, pp. 35-48, 2011.

[20] B. Yolken and N. Bambos, "Game based capacity allocation for utility computing environments," Telecommunication Systems, vol. 47, pp. 165-181, 2011.

[21] M. Perc and A. Szolnoki, “Coevolutionary games-a mini review,” BioSystems, vol. 99, no. 2, pp. 109125, 2010. 


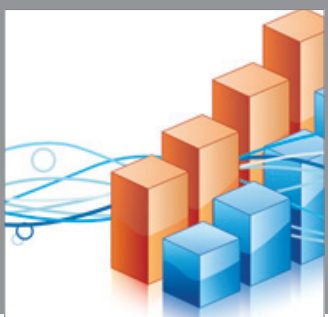

Advances in

Operations Research

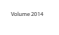

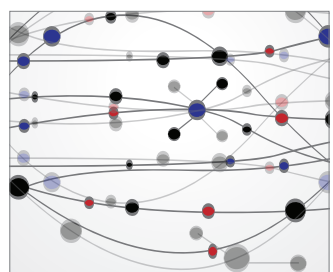

\section{The Scientific} World Journal
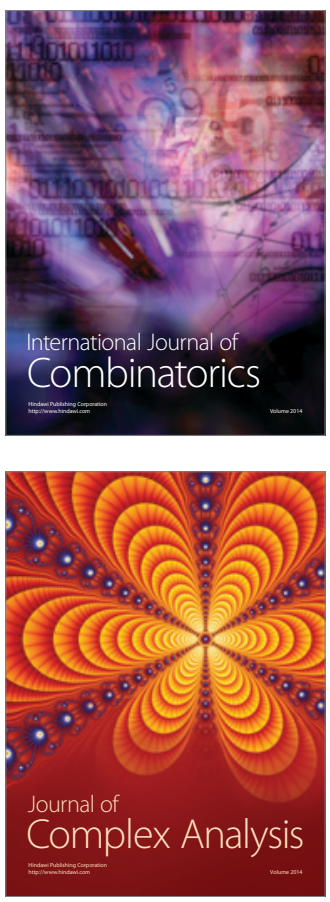

International Journal of

Mathematics and

Mathematical

Sciences
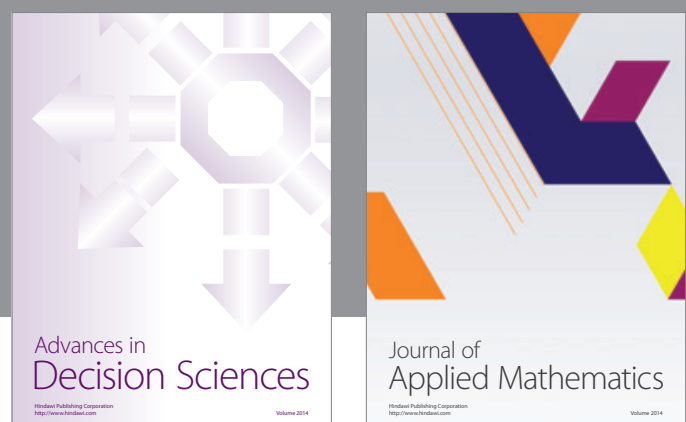

Journal of

Applied Mathematics
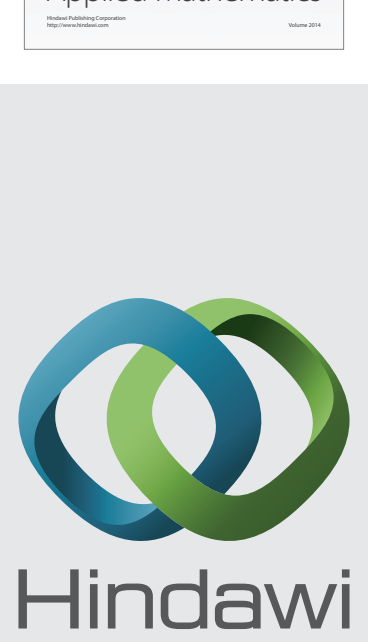

Submit your manuscripts at http://www.hindawi.com
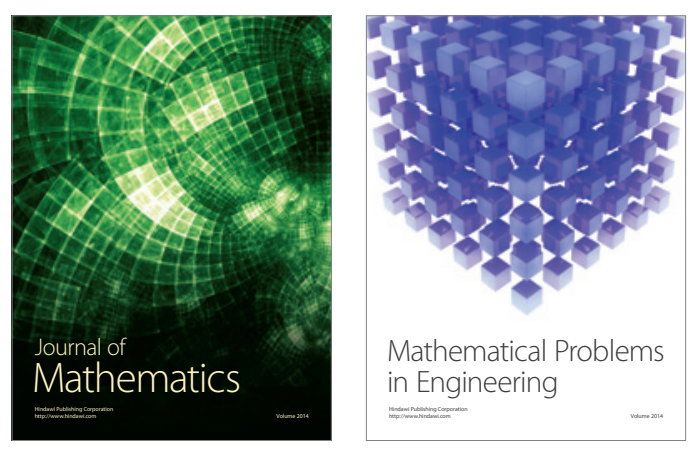

Mathematical Problems in Engineering
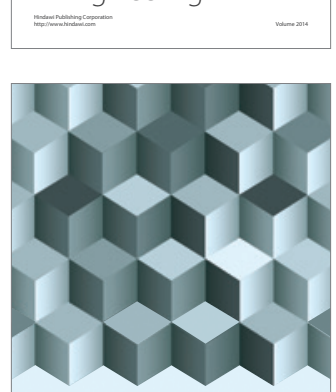

Journal of

Function Spaces
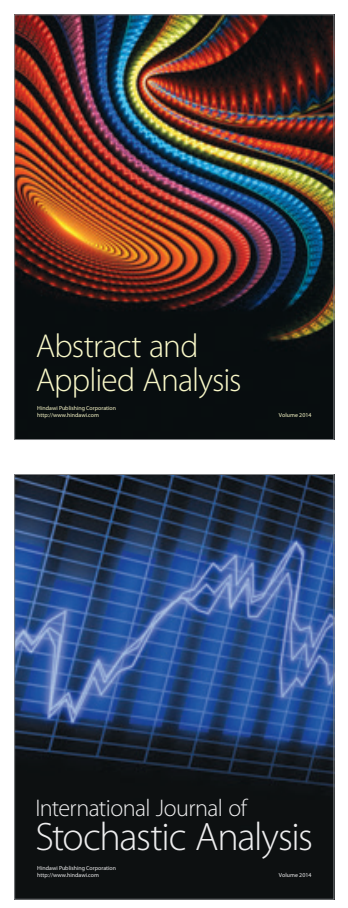

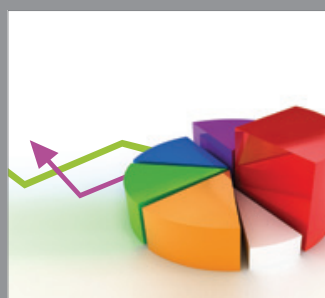

ournal of

Probability and Statistics

Promensencen
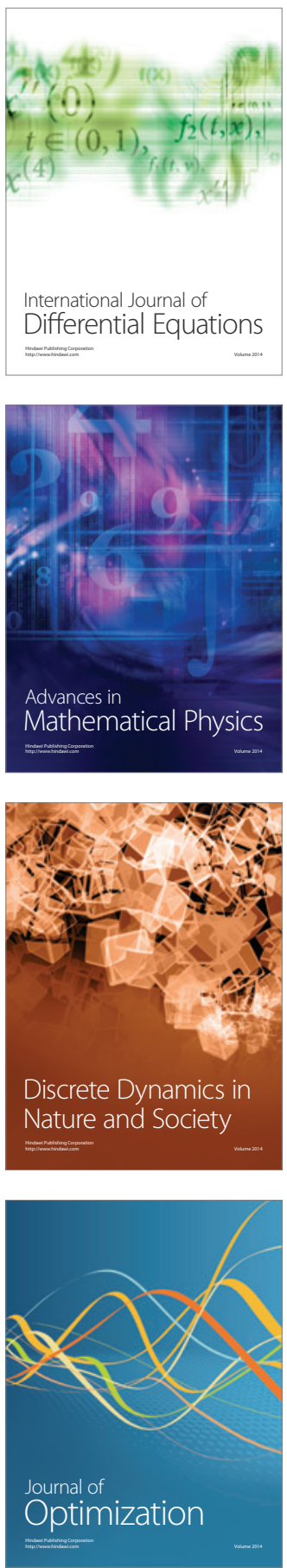